\title{
Exciton-phonon droplets with Bose-Einstein condensate: transport and optical properties
}

\author{
D. Roubtsov ${ }^{1}$, Y. Lépine ${ }^{1}$, I. Loutsenko ${ }^{2}$ \\ 1 GCM et Département de physique, Université de Montréal, C.P. 6128, succ. Centre-ville, Montreal, P.Q., H3C 3J7, Canada \\ ' ${ }^{2}$ Lockheed Martin Canada et CRM, Université de Montréal, C.P. 6128, succ. Centre-ville, Montreal, P.Q., H3C 3J7, Canada
}

\begin{abstract}
We discuss the possibility for a moving droplet of excitons and phonons to form a coherent state inside the packet. We describe such an inhomogeneous state in terms of Bose-Einstein condensation and prescribe it a macroscopic wave function. Existence and, thus, coherency of such a Bosecore inside the droplet can be checked experimentally by letting two moving packets to interact.
\end{abstract}

\section{Introduction}

Nowadays, there is a lot of experimental evidence that excitons in semiconducting crystals and heterostructures can form a strongly correlated state. In some cases, it can be assigned to the excitonic Bose-Einstein condensate (BEC) [1]. As a rule, the excitons are prepared in the ground state with $\langle\hbar \mathbf{k}\rangle \simeq 0$ (or they are in a quasiequilibrium state with some $T^{*}(t)$ cooling down toward the ground state). The conclusion about the presence of Bose-Einstein correlations among them is based on unusual properties of the direct PL signal from the excitonic cloud [1].

This article is motivated by experimental data on the transport properties of excitons in 3D crystals, such as $\mathrm{Cu}_{2} \mathrm{O}$ [2], and 2D sheets in $\mathrm{BiI}_{3}$ [3]. It is important to "cook" the following initial conditions: a relatively dense cloud of excitons with the density of $n_{\mathrm{x}}>n_{c}(T)$ (e.g., $n_{c}(T=2 \mathrm{~K}) \approx 8.7 \times 10^{16} \mathrm{~cm}^{-3}$ ) to be in a moving state with $\left\langle\hbar \mathbf{k}_{\mathrm{x}}\right\rangle \neq 0$. In the case of $\mathrm{Cu}_{2} \mathrm{O}$ crystals, these conditions can be achieved because there is approximately the same number of long wavelength acoustic phonons being formed in the same place as the excitons, $N_{\mathrm{ph}} \simeq N_{\mathrm{x}}$ and $\varepsilon_{\mathrm{ph}} \simeq 1-5 \mathrm{meV}$. Due to the excitonphonon interaction and quasi-1D geometry of the initial conditions, the acoustic phonons and excitons can form a packet with $\left\langle\hbar \mathbf{k}_{\mathrm{ph}}\right\rangle \neq 0$ and $\left\langle\hbar \mathbf{k}_{\mathrm{x}}\right\rangle \neq 0$, so that

$\left\langle\hbar \mathbf{k}_{\mathrm{x}}\right\rangle\left\|\left\langle\hbar \mathbf{k}_{\mathrm{ph}}\right\rangle\right\| O x$

and $\varepsilon_{\mathrm{x}}=m_{\mathrm{x}} v^{2} / 2<m_{\mathrm{x}} c_{\mathrm{s}}^{2} / 2 \sim 10^{-3} E_{\mathrm{x}}$. Here, $c_{\mathrm{s}}=$ $4.5 \times 10^{5} \mathrm{~cm} / \mathrm{s}$ is the (longitudinal) speed of sound, and $E_{\mathrm{x}} \simeq 0.15 \mathrm{eV}$ is the exciton Rydberg, and $m_{\mathrm{x}} \simeq 1.5 m_{\mathrm{e}}$ is the exciton mass.
We assume that a Bose-correlated exciton-phonon core can be formed inside the exciton-phonon packet under these conditions. Then, such a packet can move ballistically through the whole crystal at $T<T_{c}$, and the coherency of the Bose-core can be revealed by the packetpacket interaction or by stimulated scattering into the packet with the condensate. If $T>T_{c}$ or $n_{\mathrm{x}}<n_{c}(T)$, the exciton-phonon packet exhibits the standard diffusive behavior [2]. Thus, the $3 \mathrm{D}$ droplets that supposed to contain the excitonic Bose-Einstein condensate are found in a spatially inhomogeneous state with the well-defined characteristic width $L_{\mathrm{ch}}$ in the direction of motion. Note that this width $L_{\mathrm{ch}}=c_{\mathrm{s}} \tau_{\mathrm{ch}}$ can be different from the Bose correlation length of the Bose-core, $L_{0}=c_{\mathrm{S}} \tau_{0}$. For example, the estimate $L_{0} \sim 10^{-1} L_{\mathrm{ch}}$, or

$\tau_{0} \simeq 50-60 \mathrm{~ns} \sim 10^{-1} \tau_{\mathrm{ch}} \simeq 0.2-0.4 \mu \mathrm{s}$,

can be extracted from the experiments on two packet interaction at $T<T_{c}$ 2]. The registered ballistic velocities of such excitonic packets turn out to be always less, but relatively close to the longitudinal sound speed of the crystal, $v<c_{\mathrm{s}}$. Note that the paraexcitons in pure $\mathrm{Cu}_{2} \mathrm{O}$ crystals have an extremely large lifetime, $\tau \gg 13 \mu \mathrm{s}$, and a moving exciton with $\hbar k_{x} \sim m_{\mathrm{x}} c_{\mathrm{s}}$ cannot be converted into a photon directly. Then, one can exclude the photons from simple models describing the transport of a single packet of excitons in a periodic medium. Moreover, we neglect the ortho-para exciton conversion inside the formed packet of the moving paraexcitons, (i.e., $N_{\mathrm{x} \text {, para }}>N_{c}, N_{\mathrm{x} \text {, ortho }} \simeq 0$ ) in $\mathrm{Cu}_{2} \mathrm{O}$ crystals [4].

To understand the physics of anomalous excitonic transport, we assume that the macroscopic wave function $\Psi_{0} \simeq \phi_{\mathrm{o}} e^{i \varphi_{\mathrm{c}}}$ can be associated with the coherent part of the excitonic packet at $T<T_{c}$. Here, $\varphi_{\mathrm{c}}$ is the coherent phase of the condensate. Indeed, the experimental results [2], 3] suggest the following decomposition of the density of excitons in the packet,

$n(\mathbf{x}, t)=n_{\mathrm{coh}}(\mathbf{x}, t)+\Delta n(\mathbf{x}, t)$, 
where $n_{\text {coh }}(\mathbf{x}, t) \approx n_{\text {core }}(x-v t)$ is the ballistic (superfluid) part of the packet,

$n_{\text {core }}(x-v t) \simeq\left|\Psi_{0}\right|^{2}(x-v t)$,

and $\Delta n(\mathbf{x}, t)$ is the noncondensed part of it. The following decomposition can be written for the out-of-condensate part:

$$
\Delta n(\mathbf{x}, t)=\left\langle\delta \hat{\psi}^{\dagger} \delta \hat{\psi}(\mathbf{x}, t)\right\rangle \approx \delta n_{\text {cloud }}(\mathbf{x}, t)+\delta n_{\text {tail }}(\mathbf{x}, t) .
$$

The challenging problem is how to describe the spatially inhomogeneous state of the droplet with the excitonic BEC inside in terms of $\Psi_{0}(\mathbf{x}, t)$ and $\delta \hat{\psi}(\mathbf{x}, t)$, where $\delta \hat{\psi}$ is the "fluctuating" part of the exciton Bose-field. For example, within the quasistationary approximation, one has to calculate $n_{\text {core }}\left(x / L_{0}\right)$ and

$$
\Delta n(x) \simeq \delta n_{\mathrm{o}, \text { cloud }}\left(x / L_{\mathrm{ch}}\right)+\delta n_{\text {tail }}
$$

and understand how the different characteristic lengthes and coherence properties appear in the theory with the Bose-condensate. Note that if the excitonic packet moves in a crystal (or another semiconductor structure), it interacts with thermal phonons, noncondensed excitons, impurities and other imperfections of the lattice, etc.. Then, the coherent core of the packet can be found in a quasi-stable state, and the fluctuations of $\phi_{\mathrm{o}}(x-v t)$ and, especially, $\varphi_{\mathrm{c}}(x, t)$ can be of a great importance for possible experimental verifications of their existence.

\section{Exciton-Phonon Condensate}

To obtain the necessary density of excitons $n_{\mathrm{x}}$ in the excitonic cloud and, thus, meet the BEC conditions, the crystals are irradiated by laser pulses with $\hbar \omega_{L} \gg E_{\text {gap }}$, and the temperature of the crystal is $T \simeq 1 \sim 5 \mathrm{~K}$. If the cross-section area $S$ of an excitation spot on the surface of the crystal can be made large enough, such as $S \approx S_{\text {surf }}$, the hot droplet of paraexcitons can acquire an average momentum during its thermolization process $\left(T^{*}(t) \rightarrow T\right)$. Indeed, the phonon wind, or the flow of nonequilibrium phonons, blows unidirectionally from the surface into the bulk [5] and transfers the nonzero momentum to the excitonic cloud,

$\mathbf{P}_{\mathrm{x}} \simeq N_{\mathrm{x}}\left\langle\hbar \mathbf{k}_{0}\right\rangle \neq 0$ and $\mathbf{P}_{\mathrm{x}} \perp S_{\text {surf }}$,

see Fig. 1. As a result, the packet of moving excitons and nonequilibrium phonons of the phonon wind $\left(N_{\mathrm{ph}} \simeq N_{\mathrm{x}}\right)$ is actually the system that undergoes the transition toward developing the Bose-Einstein correlations at $T^{*}<$ $T_{c}$.

Let us assume that the condensate has been already formed inside the moving excitonic droplet, and the following representation of the exciton Bose-field holds: $\hat{\psi}=\Psi_{0}+\delta \hat{\psi}$. For the displacement field of the crystal $\hat{\mathbf{u}}$, we introduce a nontrivial coherent part too, i.e., $\hat{\mathbf{u}}=$ $\mathbf{u}_{0}+\delta \hat{\mathbf{u}}, \mathbf{u}_{0} \neq 0$. The important property of the excitonphonon condensate is a kind of the self-consistency condition, roughly,

$\partial \mathbf{u}_{0}(x-v t) \propto\left|\Psi_{0}(x-v t)\right|^{2}$.

In these terms, the moving packet contains both the macroscopically occupied exciton-phonon condensate, or

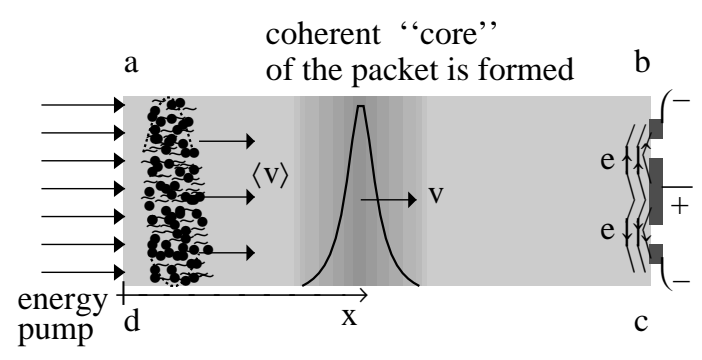

Fig. 1 A medium, in which the exciton-phonon droplet can propagate, is presented in the form of the channel 'abcd' on this Figure. After some amount of energy has been pumped into the medium during a short time interval and absorbed near a boundary, a localized excited state is formed near the face 'ad'. If there is a mechanism of the momentum transfer to the excited state, the droplet begins to move toward the opposite face 'bc' with the velocity $\langle v\rangle$. Then, such conditions can favor the appearance of an inhomogeneous coherent state inside the droplet if the average density of the excitons $n_{\mathrm{x}}>n_{c}(T)$. The profile of the excitonic part of it, $n_{\text {core }}(x, t) \simeq\left|\Psi_{\mathrm{o}}(x, t)\right|^{2}$, is shown by the bold line and the intensity of the elastic (phonon) part, $\partial_{x} u_{\mathrm{o}, x}(x, t)$, is represented by changements of the intensity of the background color. When the packet reaches the face 'bc', the total density of excitons $n(x)$ is converted into an electric current, $i(t)$.

the Bose-core $\Psi_{0}(\mathbf{x}, t) \cdot \mathbf{u}_{0}(\mathbf{x}, t)$, and out-of-condensate excitons and phonons. The macroscopic wave function of excitons $\Psi_{0}(x, t)$ is normalized as follows:

$\int\left|\Psi_{0}\right|^{2}(x, t) d \mathbf{x}=S \int \phi_{\mathrm{o}}^{2}\left(x / L_{0}\right) d x=N_{\mathrm{o}} \gg 1$,

where $N_{\mathrm{O}}$ is the macroscopic number of condensed excitons, and, generally, $N_{\mathrm{o}}(T)<N_{\mathrm{x}}$. Within the quasistationary approximation, we have $N_{\mathrm{o}}(T)=$ const and $\delta N(T)=$ const that implies (quasi)stability of the moving packet with the Bose-core during the finite observation time.

To model the ballistic motion of a single packet, we use the following ansatz for the Bose-core of the packet: $\Psi_{0}(x, t)=\mathrm{e}^{-i\left(\tilde{E}_{g}+m_{\mathrm{x}} v^{2} / 2-|\mu|\right) t} \mathrm{e}^{i\left(\varphi_{\mathrm{c}}+k_{0} x\right)} \phi_{\mathrm{o}}(x-v t)$,

$u_{0 j}(x, t)=u_{\mathrm{o}}(x-v t) \delta_{1 j}$,

where $\tilde{E}_{g}=E_{\text {gap }}-E_{\mathrm{x}}, \varphi_{\mathrm{c}}=$ const is the macroscopic phase, $\hbar k_{0}=m_{\mathrm{x}} v$, and $\mu=\mu\left(N_{\mathrm{o}}\right)<0$ is the effective chemical potential of the condensate. At $T \ll T_{c}$, one can disregard the interaction between the Bose-core and the out-of-condensate cloud and write down the following equations on the envelope functions $\phi_{\mathrm{o}}\left(x / L_{0}\right)$ and $u_{\mathrm{o}}\left(x / L_{0}\right)[6]:$

$-|\mu| \phi_{\mathrm{o}}(x)=-\left(\hbar^{2} / 2 m_{\mathrm{x}}\right) \partial_{x}^{2} \phi_{\mathrm{o}}(x)-\left|\tilde{\nu}_{0}\right| \phi_{\mathrm{o}}^{3}(x)+\tilde{\nu}_{1} \phi_{\mathrm{o}}^{5}(x)$,

$\partial_{x} u_{\mathrm{o}}(x) \approx-$ const $_{0} \phi_{\mathrm{o}}^{2}(x)+\operatorname{const}_{1} \phi_{\mathrm{o}}^{4}(x)$.

At $T \neq 0, T<T_{c}$, we choose the quasistationary approximation to write out the decomposition of the exciton and phonon fields of the moving droplet,

$\hat{\psi}_{0}(\mathbf{x}, t)=\exp \left(i \varphi_{c}(x, t)\right)\left\{\phi_{\mathrm{o}}(x-v t)+\delta \hat{\psi}_{\mathrm{o}}\left(x-v t, \mathbf{x}_{\perp}, t\right)\right\}$, $\hat{u}_{0, j}(\mathbf{x}, t)=u_{\mathrm{o}}(x-v t) \delta_{1, j}+\delta \hat{u}_{\mathrm{o}, j}\left(x-v t, \mathbf{x}_{\perp}, t\right)$. 
Then, the following correlation functions have to be included into an analog of Eq. (10): the 'anomalous' ones, such as $\tilde{\mathrm{m}}(x)=\left\langle\delta \hat{\psi}_{\mathrm{o}} \delta \hat{\psi}_{\mathrm{o}}\right\rangle$, the exciton-phonon correlators, such as $\tilde{q}_{j}=\left\langle\partial_{j} \delta \hat{u}_{\mathrm{o}, j} \delta \hat{\psi}_{\mathrm{o}}\left(x, \mathbf{x}_{\perp}, t\right)\right\rangle$, and the outof-condensate density of the excitons and phonons,

$\delta n_{\mathrm{o}}(x)=\left\langle\delta \hat{\psi}_{\mathrm{o}}^{\dagger} \delta \hat{\psi}_{\mathrm{o}}\left(x, \mathbf{x}_{\perp}, t\right)\right\rangle$ and $Q_{x x}(x)=\left\langle\left(\partial_{x} \delta \hat{u}_{\mathrm{o}, x}\right)^{2}\right\rangle$.

It is possible to generalize Eq. (10) to the case of $T \neq 0$. Here, we write it in the following (tractable) form:

$$
\begin{aligned}
& -(|\mu|+\delta \mu) \phi_{\mathrm{o}}(x)=-\left(\hbar^{2} / 2 m^{*}\right) \partial_{x}^{2} \phi_{\mathrm{o}}(x)+\left(\tilde{\nu}_{0}+\delta \nu_{0}\right) \phi_{\mathrm{o}}^{3}(x) \\
& +\left(\tilde{\nu}_{1}+\delta \nu_{1}\right) \phi_{\mathrm{o}}^{5}(x), \\
& \partial_{x} u_{\mathrm{o}}(x) \approx-\operatorname{const}_{0}^{\prime} \phi_{\mathrm{o}}^{2}(x)+\operatorname{const}_{1}^{\prime} \phi_{\mathrm{o}}^{4}(x)-\mid \text { const }_{\text {tail }} \mid .
\end{aligned}
$$

To a first approximation, we disregard all the dissipation terms and assume $T^{*} \simeq T$. Then, the corrections to the effective chemical potential $|\mu|$, the $\mathrm{x}-\mathrm{x}$ and $\mathrm{x}-$ ph interaction vertices, $\tilde{\nu}_{j}$ and const $_{j}$, depend on the temperature $T$ through the above mentioned correlation functions. This means Eq. (12) has to be solved together with the equations on the out-of-condensate excitons and phonons, $\delta \hat{\psi}_{\mathrm{o}}, \delta \hat{\psi}_{\mathrm{o}}^{\dagger}$, and $\delta \hat{u}_{\mathrm{o}, j}$. Note that at $T \neq 0, T<T_{c}$ the effective interaction vertices in Eq. (12) are strongly renormalized in comparison with the "bare" ones staying in the Hamiltonian of the excitonphonon system. For example, the localized solution for

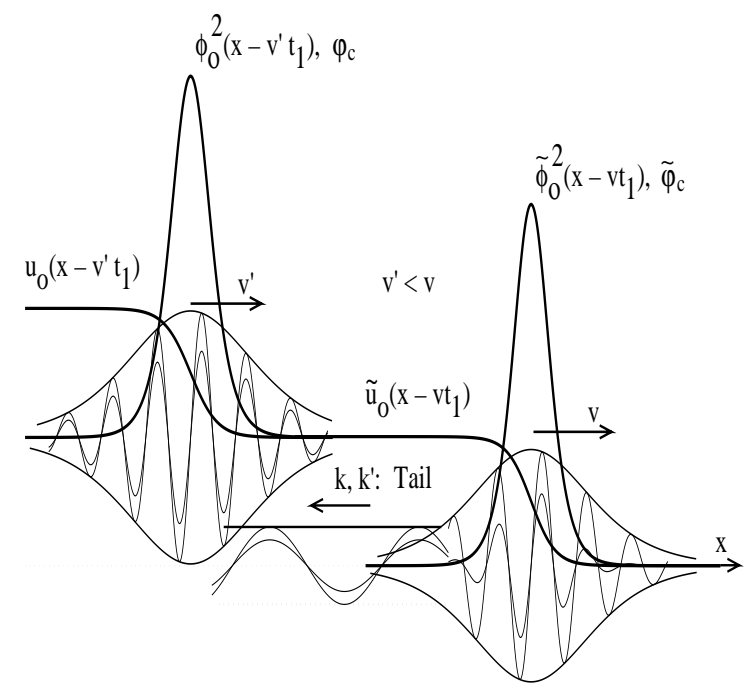

Fig. 2 Two ballistic packets with two exciton-phonon condensates $\left(\mathrm{e}^{i \varphi_{c}(x, t)} \phi_{\mathrm{o}}(x-v t) \cdot u_{\mathrm{o}}(x-v t) \delta_{1 j}\right)$ inside were created with the same concentration of excitons and the same ballistic velocity, $v$, in a crystal. The time delay between them is a free parameter. Then, two different interaction regimes are possible. The first one corresponds to the case in which the Bose-cores of the packets overlap. This is a strong interaction case, and the packets can merge into one droplet. The second regime, in which the Bose-cores do not overlap, is the case of weak interaction between the packets. It is depicted on this Figure. However, the second moving packet (the left one) can "feel" the first packet (the right one) through the interaction with the exciton-phonon tail of the first one. As a result, the second packet slows down, $v^{\prime}<v$, and becomes more broad. $\phi_{\mathrm{o}}\left(x / L_{0}\right)$ (a kind of the "bright" soliton of the cubicquintic NLS equation) exists due to the effect of such a strong renormalization, $\tilde{\nu}_{0}+\delta \nu_{0}<0$ and $\tilde{\nu}_{1}+\delta \nu_{1}>0$.

Note that the dependence of $n_{\mathrm{o}}\left(x / L_{\mathrm{o}}\right)$ on $N_{\mathrm{o}}$ is highly nonlinear. As a rough estimate, we can write $n_{\mathrm{o}} \propto N_{\mathrm{o}}^{2}$ $(T \rightarrow 0)$. This suggests that the estimates presented in [7] have to be revisited. Some qualitative results obtained for the coherent exciton-phonon packets are presented on Fig. 2.

Finally, we speculate on the possibility to convert the coherent exciton field into the coherent photon one by colliding two moving exciton-phonon packets. Such an experiment could reveal how rigid is the macroscopic phase of the Bose-core (if any). If one can prescribe a macroscopic phase $\varphi_{c}$ to each Bose-core of the moving packets, the face-to-face collision of them could result in the many-photon production. For example, the "jets" of phonons originated from the condensate interaction (the core-to-core collision) seem to be highly directional in space and with a low noise level, see Fig. 3.

\section{References}

1. J. L. Lin, J. P. Wolfe, Phys. Rev. Lett. 71, (1993) 122;

L.V. Butov, A. I. Filin, Phys. Rev. B 58, (1998) 1980;

T. Goto, M. Y. Shen et al., Phys. Rev. B 55, (1997) 7609.

2. E. Fortin, S. Farad, and A. Mysyrowicz, Phys. Rev. Lett. 70, (1993) 3951;

A. Mysyrowicz, E. Benson, and E. Fortin, Phys. Rev. Lett.

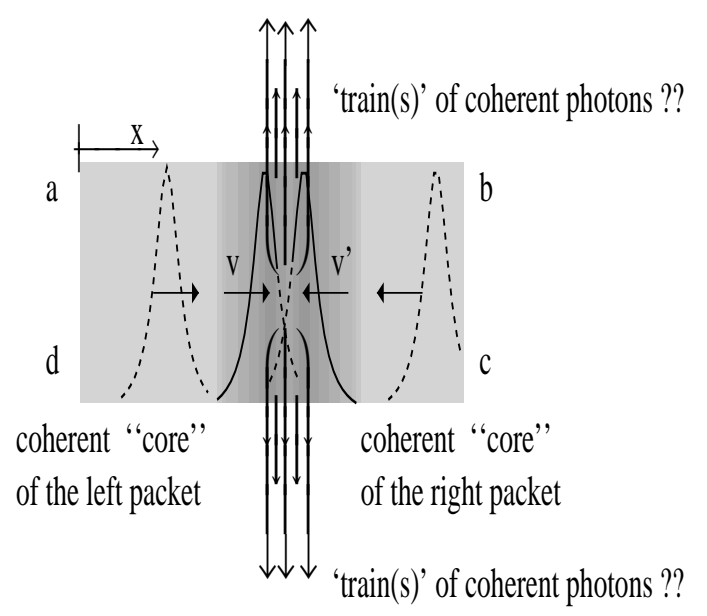

Fig. 3 After some amount of energy $\left(\delta E_{\text {left }}=\delta E_{\text {right }}\right)$ has been pumped into the medium during a short time interval $\delta t$ and absorbed near the left and right boundaries, two localized excited states are formed near the faces 'ad' and 'bc'. If there is a mechanism of the momentum transfer to the excited state, the droplets begin to move toward the opposite faces with the velocities $\langle v\rangle \approx\left\langle v^{\prime}\right\rangle$. Such conditions can favor the appearance of a coherent boson-phonon state (an analog of Davydov soliton) inside both the left and right droplets. The collision of such exciton-phonon droplets seems to be analogous to the heavy nucleus collisions that result in the production of pion jets. In $\mathrm{Cu}_{2} \mathrm{O}$ crystal, one can expect the production of two photon "jets", and coherence of the phonons in such jets can be checked experimentally. 
77, (1996) 896;

E. Benson, E. Fortin et al., Europhys. Lett. 40, (1997) 311;

E. Fortin, E. Benson, A. Mysyrowicz, Electrochem. Soc. Proceed. 98-25, (1998) 1.

3. H. Kondo, H. Mino et al., Phys. Rev. B 58, (1998) 13835.

4. K. E. O'Hara, J. R. Gullingsrud, and J. P. Wolfe, Phys. Rev. B 60, (1999) 10 872;

G. M. Kavoulakis, A. Mysyrowicz, Phys. Rev. B 61, (2000) 16619.

5. G. A. Kopelevich, S. G. Tikhodeev, and N. A. Gippius, JETP 82, (1996) 1180;

S. G. Tikhodeev, N. A. Gippius, and G. A. Kopelevich, Phys. Stat. Sol. (a) 178, (2000) 63.

6. D. Roubtsov, Y. Lépine, Phys. Rev. B 61, (2000) 5237; see also in cond-mat archive at http://xxx.lanl.gov/.

7. S. G. Tikhodeev, Phys. Rev. Lett. 78, (1997) 3225. 